\title{
Caracterización funcional de plantas y su utilidad en la selección de especies para la restauración ecológica de ecosistemas altoandinos
}

\author{
Functional characterization of plants and their utility in the \\ selection of species for the ecological restoration of \\ high-Andean ecosystems
}

\author{
Angélica María Cogollo Calderón ${ }^{(1)}$, Patricia Velasco Linares ${ }^{(0)}$, Leonardo Manosalva ${ }^{(0)}$
}

\section{Resumen}

Para iniciar un proceso de restauración en áreas disturbadas, es importante seleccionar especies vegetales nativas que aporten diversidad funcional al ecosistema en recuperación y permitan el establecimiento de nuevos individuos hacia etapas sucesionales más avanzadas. Evaluamos 14 rasgos de historia de vida en 20 especies de una zona de transición entre bosque altoandino y páramo en la reserva Aguas Vivas, Soacha, Colombia. Registramos información de tipos de polinización y dispersión, hábito de crecimiento, altura máxima, tipo y tamaño del fruto, número de semillas por fruto, textura de la hoja, tipo de indumento, área foliar, área foliar específica, nitrógeno foliar, contenido foliar de materia seca y dureza foliar. El análisis de componentes principales mostró que el nitrógeno foliar, la dureza foliar y el área foliar específica fueron los rasgos más informativos para definir asociaciones entre especies. Encontramos tres tipos funcionales que comprenden plantas con diferentes estrategias de adaptación, que se considerarán a la hora de seleccionar grupos de especies vegetales a reintroducir en ambientes modificados. Sin embargo, esta selección también se debe realizar teniendo en cuenta el conocimiento local, la disponibilidad de propágulos, y el estado sucesional de las áreas en recuperación.

Palabras clave. Disturbios. Grupo Funcional. Rasgos de historia de vida. Subpáramo. Sucesión vegetal.

\begin{abstract}
To start a restoration process in disturbed areas, it is important to select plant species that provide functional diversity to the recovering ecosystem and allow the establishment of new individuals towards more advanced stages of succession. We evaluated 14 life history traits in 20 species in a transition zone between high Andean forest and paramo in the Aguas Vivas reserve, Soacha, Colombia. We record information on pollination and dispersal types, growth habit, maximum height, fruit type and size, number of seeds per fruit, leaf texture, leaf area, specific leaf area, leaf nitrogen, leaf dry matter content and leaf hardness. The principal components analysis showed that leaf nitrogen, leaf hardness and specific leaf area were the most informative features to define associations between species. We found three functional types that include plants with different adaptation strategies, which will be considered when selecting plant species to be introduced in modified environments. However, this selection should also be made taking into account local knowledge, availability of propagules, and the successional status of the recovering areas.
\end{abstract}

Key word. Disturbance. Functional group. Life history traits. Subparamo. Plant succession. 


\section{Introducción}

Los bosques altoandinos se consideran "hotspot" de biodiversidad y encabezan la lista de las áreas más vulnerables a nivel mundial, principalmente por las altas tasas de deforestación y el desarrollo de proyectos que influyen en la transformación acelerada del territorio (Mittermeier \& Mittermeier, 1997; Kappelle \& Brown, 2001). En Colombia, una buena parte de la diversidad florística se alberga en ecosistemas de alta montaña (Van der Hammen \& González, 1960); sin embargo, su pérdida impacta negativamente la riqueza de especies $\mathrm{y}$, por tanto, la diversidad funcional y servicios ecosistémicos que estos brindan (Anderson et al., 2011).

Debido a la oferta hídrica que aportan las áreas rurales del Distrito Capital de Bogotá, es necesario desarrollar estrategias que prioricen la conservación y restauración ecológica de zonas degradadas (Van der Hammen et al., 2008), donde se incluyan especies vegetales con características que reactiven procesos de regeneración natural y posibiliten la restitución de las funciones del sistema y sus características florísticas y estructurales (Naeem et al., 1994; Hooper \& Vitousek, 1997). Esta selección se puede simplificar agrupando las especies según sus rasgos funcionales (Castellanos-Castro \& Bonilla, 2011; Montenegro \& Vargas, 2008; Meli et al., 2014), ya que generalmente las especies adaptadas a ambientes con frecuentes disturbios, ya sean naturales o antrópicos, desarrollan ciertos atributos que contribuyen a la recuperación de áreas disturbadas (Holl, 2002). Además, esta agrupación funcional se considera una herramienta adecuada para el diagnóstico y planteamiento de procesos de restauración ecológica (Kooyman \& Rossetto, 2008) y para la conservación en términos de la selección de especies a introducir en ambientes modificados (Castellanos-Castro \& Bonilla, 2011).

El objetivo de esta investigación fue la evaluación de rasgos funcionales relacionados a la historia de vida de 20 especies en un sitio de transición entre bosque altoandino y páramo, afectado por la expansión de la frontera agropecuaria, y de esta manera contribuir en la identificación de tipos funcionales importantes en la recuperación de zonas alteradas en ecosistemas de alta montaña.

\section{Materiales y métodos}

Área de estudio. La investigación se llevó a cabo en el predio Aguas Vivas, entre los límites de la vereda Hungría en el municipio de Soacha y la vereda Quiba Alta de Bogotá (coordenadas: $4^{\circ} 28.638^{\prime} \mathrm{N}, 74^{\circ} 10.94^{\prime} \mathrm{O} ; 3330$ m s. n. m.), durante el periodo comprendido entre los meses de junio de 2013 a junio de 2014. Este predio fue adquirido por la Administración Municipal de Soacha, para preservar la riqueza hídrica de la zona y contribuir con la recuperación de áreas degradadas por uso agropecuario. Cuenta con un área de 97.61 ha de las cuales un $50 \%$ corresponde a coberturas de pastos exóticos donde se evidencia un proceso de sucesión alterada o detenida y el otro porcentaje restante a coberturas naturales de páramo y subpáramo en un mejor estado de conservación. La reserva es de gran importancia, puesto que aquí se encuentra el nacimiento del río Soacha.

Caracterización vegetal. Se trazaron cinco parcelas de $50 \mathrm{~m} \times 2 \mathrm{~m}\left(100 \mathrm{~m}^{2}\right)$, en las que se registró información para todos los individuos de porte arbustivo y arbóreo con alturas (h) mayores a $1 \mathrm{~m}$ y diámetro a la altura del pecho (DAP) superior a $1 \mathrm{~cm}$. Se recolectaron datos de h, DAP y cobertura de los individuos. Para la vegetación herbácea se hicieron diez muestreos en cada parcela con ayuda de un cuadrante de $1 \mathrm{~m}^{2}$ que estaba subdividido en 100 cuadrados de $10 \mathrm{~cm}^{2}$ (para facilitar la estimación de la cobertura), y en cada muestreo se midió la altura promedio con ayuda de un flexómetro y el porcentaje de cobertura de todas las especies con altura menor a $1 \mathrm{~m}$. Se recolectó el material vegetal para su posterior determinación y se depositó en el herbario del Jardín Botánico José Celestino Mutis (JBB; http:/ / colecciones.jbb.gov.co/herbario).

Selección de las especies. Se escogieron las especies dominantes según el Índice de Valor de Importancia (IVI), el cual se calcula para cada especie a partir de la suma de la abundancia relativa, la frecuencia relativa y la dominancia relativa (Curtis \& McIntosh, 1951).

Selección de rasgos funcionales. Los mejores rasgos son aquellos que desde el punto de vista ecológico ayudan a superar las limitaciones bióticas y abióticas a la regeneración natural (disponibilidad de propágulos, 
condiciones climáticas, oferta hídrica y de nutrientes, entre otros) y pueden ser medidos en un menor tiempo, a un menor costo y más fácilmente, para un número representativo de individuos dentro de una población (Cornelissen et al., 2003). Con base en esto, se seleccionaron algunos rasgos asociados a la fase de dispersión, establecimiento y persistencia en especies vegetales, según lo propuesto por Weiher et al. (1999), Lavorel et al. (1998), Díaz \& Cabido (1997) (Tabla 1).

Obtención de los datos. Para la evaluación de los rasgos foliares se seleccionaron cinco individuos por especie.
Por cada individuo adulto se recolectó una muestra aleatoria en promedio de cinco hojas maduras completamente desarrolladas, sin evidencia de daños por patógenos ni herbivoría, siguiendo el protocolo para la toma de muestras foliares de Garnier et al. (2001).

El área foliar (AF) se determinó escaneando las hojas frescas a través de un escáner marca HP psc 1310. Después de esto, las hojas se secaron durante 48 horas en un horno a $70^{\circ} \mathrm{C}$ y se pesaron, para luego determinar el área foliar específica (AFE) (promedio del área foliar/ promedio del peso seco) por medio del software libre ImageJ (Rasband, 2007).

Tabla 1. Rasgos funcionales de plantas asociados a la fase de dispersión, establecimiento y persistencia

\begin{tabular}{|c|c|c|}
\hline FASE & RASGOS & FUNCIÓN \\
\hline \multirow{5}{*}{ Dispersión } & Tipo de fruto & \multirow{5}{*}{$\begin{array}{l}\text { Relacionado con la capacidad para colonizar o prosperar } \\
\text { en un hábitat (Rozendaal et al., 2006) }\end{array}$} \\
\hline & Tamaño del fruto & \\
\hline & Número de semillas por fruto & \\
\hline & Estrategia de dispersión & \\
\hline & Estrategia de polinización & \\
\hline \multirow{4}{*}{ Establecimiento } & Área Foliar Específica, AFE $\left(\mathrm{cm}^{2} \cdot \mathrm{g}^{-1}\right)$ & $\begin{array}{l}\text { Íntimamente relacionado a la capacidad fotosintética } \\
\text { (Kitajima, 1996) y el balance del carbono en la planta, por } \\
\text { lo tanto correlacionado positivamente con el crecimiento } \\
\text { de la planta (Rozendaal et al., 2006) }\end{array}$ \\
\hline & Área Foliar, AF $\left(\mathrm{cm}^{2}\right)$ & $\begin{array}{l}\text { Fisiológicamente clave; estrechamente ligada a la distri- } \\
\text { bución de las plantas } \\
\text { A mayor área foliar, mayor inversión en estructura } \\
\text { (Cornelissen et al., 2003; Niinemets et al., 2007; Niklas } \\
\text { et al., 2007) }\end{array}$ \\
\hline & Contenido de Nitrógeno foliar, N (\%) & $\begin{array}{l}\text { Relacionado negativamente con longevidad foliar, de- } \\
\text { fensa de la hoja, potencial de crecimiento, palatabilidad } \\
\text { para herbívoros (Poorter \& Bongers, 2006) }\end{array}$ \\
\hline & Tipo de pubescencia & $\begin{array}{l}\text { Reduce evapotranspiración (Granados-Sánchez } \\
\text { et al., 1998), protección física contra herbivoría (Karban } \\
\text { \& Baldwin, 1997), reserva de sustancias lipídi- } \\
\text { cas, flavonides y compuestos fenólicos (Nielson \& } \\
\text { Griffith, 1978). }\end{array}$ \\
\hline \multirow{3}{*}{ Persistencia } & $\begin{array}{l}\text { Altura máxima, AM (m). } \\
\text { Hábito de crecimiento (árboles, } \\
\text { arbustos) }\end{array}$ & $\begin{array}{l}\text { Fuertemente relacionado con la habilidad de competir } \\
\text { por luz, longevidad (Poorter \& Bongers, 2006) } \\
\text { Relacionado con el desarrollo de las plantas y fisonomía } \\
\text { de la vegetación }\end{array}$ \\
\hline & Contenido foliar de materia seca, CFMS (\%) & $\begin{array}{l}\text { Relacionado negativamente con potencial de crecimiento } \\
\text { y positivamente con longevidad de la hoja. }\end{array}$ \\
\hline & $\begin{array}{l}\text { Dureza de la hoja, DF }\left(\mathrm{N} \cdot \mathrm{cm}^{-2}\right) \\
\text { Textura de la hoja }\end{array}$ & $\begin{array}{l}\text { Relacionado positivamente con longevidad de la hoja } \\
\text { (Dunn et al., 1976), con su velocidad de descomposición, } \\
\text { e indicador de la relación de carbono invertido en pro- } \\
\text { tección estructural de tejidos fotosintéticos. }\end{array}$ \\
\hline
\end{tabular}


El contenido foliar de materia seca (CFMS) se realizó a través de la eliminación del agua libre por medio del calor, seguida por la determinación del peso del residuo (Batteman, 1970); la cuantificación del nitrógeno foliar (N) se hizo siguiendo la metodología de Kjeldahl (AOAC, 2000). Este procedimiento se llevó a cabo en el laboratorio de Bromatología del Jardín Botánico José Celestino Mutis.

Para la caracterización de los tipos de indumento, se recolectaron hojas maduras, que tuvieran un área foliar similar. Se observó el limbo de cada hoja por las caras adaxial y abaxial, y se identificó el tipo de indumento de cada una de las especies analizadas con la ayuda de un estereoscopio marca Nikon SMZ-1000. Para la identificación de los indumentos se utilizó el glosario Ilustrado de términos botánicos de Harris \& Harris (1994). Para medir la dureza foliar (DF), cada hoja fue sostenida con dos pinzas botánicas por el ápice y la base del limbo, y con la ayuda de un penetrómetro marca EXPOTECH referencia SHC 280, se calculó la fuerza necesaria para atravesar la zona central al lado derecho de la vena media de cada una de las hojas, calculada en newtons por centímetro cuadrado $\left(\mathrm{N} \mathrm{cm}^{-2}\right)$, método descrito por Hendry \& Grime (1993). Con los datos obtenidos se calculó el promedio y la desviación estándar para obtener los valores de DF de cada especie. La textura de la hoja se determinó con ayuda del Glosario Botánico Ilustrado (Moreno, 1984).

Para evaluar los rasgos reproductivos, se recolectaron en promedio quince frutos a partir de cinco individuos de la misma especie; estos debían estar en lo posible maduros en el caso de bayas, drupas o legumbres y secos en el caso de los aquenios. En fresco, se tomaron medidas de longitud $(\mathrm{mm})$ y diámetro $(\mathrm{mm})$ para cada uno de los frutos con un calibrador con una precisión de 0.001. Los valores obtenidos de cada fruto se promediaron para cada una de las especies. También se registró el número de semillas/fruto, las cuales se contaban en su totalidad, exceptuando los frutos de las especies que presentaran un número mayor a 50 semillas, utilizando un estereoscopio marca Nikon SMZ-1000. El tipo de fruto se determinó con base en su aspecto morfológico y la forma de crecimiento con base en la clasificación de Moreno (1984).

La información sobre estrategia de dispersión y polinización se tomó de la literatura y de datos en el campo, teniendo en cuenta la forma y estructura de la semilla y de la flor. La altura máxima se obtuvo de los levantamientos de información vegetal que se tomaron en el campo.

Identificación de tipos funcionales. Se utilizó el enfoque de tipos funcionales de plantas (TFP), el cual agrupa a las especies con funcionamiento similar, independientemente de su filogenia (Gitay et al., 1999; Kooyman \& Rosetto, 2008; Delgado, 2012). Las plantas que conforman los TFP exhiben respuestas similares frente a las condiciones ambientales y también producen efectos similares en los procesos ecosistémicos (Díaz \& Cabido, 1997).

Análisis de datos. A partir de los valores obtenidos para cada una de las variables cuantitativas se realizó la prueba de Shapiro Wilks para establecer si los datos siguen una distribución Gaussiana. Con ayuda del software PAST 7.0 (Hammer \& Harper, 2005) se realizó un análisis de los componentes principales (ACP), para la selección de los parámetros que más refuerzan el agrupamiento. El análisis busca agrupar las especies en función de las variables medidas y encontrar de manera intuitiva las causas del agrupamiento. Para el ACP solo se utilizaron variables cuantitativas continuas de rasgos foliares (AF, AFE, N, CFMS y DF), asumiendo que los datos deben cumplir con el supuesto de normalidad.

Se realizó un Análisis de Conglomerados (clúster) con el programa Statsoft, para la determinación de los tipos funcionales de plantas, TFP. Una vez realizado dicho análisis y agrupadas las especies a partir de la totalidad de rasgos funcionales evaluados, se conformaron y caracterizaron los tipos funcionales resultantes. Adicionalmente, para confirmar o rechazar la hipótesis de que los grupos de plantas resultantes del análisis clúster son distintos, se realizó un análisis discriminante (AD) a partir de la prueba de T-cuadrado de Hotelling, que permite la clasificación de dichos grupos con relación a los valores encontrados en los rasgos foliares en conjunto y las posibles correlaciones presentes en las mismas. Dados dos grupos de datos multivariados, se comparan las medias de las variables clasificadoras a través de los grupos obtenidos previamente; a partir de los valores de la función discriminante se establecen cuáles son los rasgos que más discriminan (separan) las especies en cada grupo (Hammer \& Harper, 2005). Finalmente, se realizó un análisis de correlación de Pearson entre los rasgos foliares cuantitativos (AFE, AF, CFMS, N, DF) para establecer el grado de correlación entre las cinco variables. 


\section{Resultados}

En el inventario de vegetación se encontraron 50 especies distribuidas en 22 familias. Las familias más representativas fueron Asteraceae (12 especies), Rosaceae (7 especies) y Melastomataceae (5 especies; Figura 1).

A partir del IVI, las especies con mayor dominancia fueron Bucquetia glutinosa, Pentacalia pulchella, Miconia ligustrina, Ageratina tinifolia, Miconia elaeoides y Macleania rupestris.

Para la evaluación de los rasgos de historia de vida, se seleccionaron en su mayoría especies del estrato arbóreo-arbustivo (Tabla 2), para las cuales se tuvieron en cuenta la dominancia, observaciones en campo y su utilización en procesos de restauración ecológica adelantados en el Jardín Botánico de Bogotá.

Rasgos funcionales. Para los rasgos foliares se determinó el peso seco, peso fresco, CFMS, N, AF, AFE y DF para las 20 especies (Tabla 3). La prueba de normalidad multivariante estableció una distribución normal de los valores promedio provenientes de las variables AF, AFE, N, CFMS y DF ( $\alpha>0.05$ g.l. $=35 n=100)$.

El rasgo foliar más heterogéneo fue el AF (124.12\%) que se debe principalmente a la variabilidad aportada por las especies Ageratina asclepiadea y Vallea stipularis. Los demás rasgos tuvieron una mayor homogeneidad, presentando coeficientes de variación menores al 45\%, exceptuando la dureza foliar (Tabla 4).

Los tipos de indumento en las hojas fueron glabro (Bucquetia glutinosa, Miconia ligustrina, Pentacalia pulchella, Gaiadendron punctatum, Cestrum buxifolium, Vallea stipularis, Hypericum juniperinum, Symplocos theiformis, Ageratina tinifolia, Miconia elaeoides, Berberis goudotii), manicado (Diplostephium rosmarinifolium, Morella parvifolia, Hesperomeles goudotiana), piloso (Baccharis prunifolia, Lupinus bogotensis), panoso (Myrsine dependens), lanado (Gynoxys fuliginosa) y seríceo (Pentacalia ledifolia). Las especies con mayor producción de semillas fueron las pertenecientes a las familias Melastomataceae y Asteraceae, y las de menor producción: Gaiadendron punctatum, Morella parvifolia, Myrsine dependens y Symplocos theiformis. El 35 \% de las especies presentaron frutos de tipo aquenio (Pentacalia pulchella, Ageratina asclepiadea, Diplostephium rosmarinifolium, Gynoxys fuliginosa, Baccharis prunifolia, Ageratina tinifolia, Pentacalia ledifolia), seguidas por bayas (Miconia ligustrina, Cestrum buxifolium, Miconia elaeoides, Berberis goudotii) y drupas (Gaiadendron punctatum, Morella parvifolia, Myrsine dependens, Symplocos theiformis) con 20 \% cada una; las demás especies presentan cápsulas (Bucquetia glutinosa, Vallea stipularis, Hypericum juniperinum), legumbres (Lupinus bogotensis) y pomos (Hesperomeles goudotiana). Las estrategias de dispersión que se encontraron fueron balocoria, barocoria, zoocoria y anemocoria.

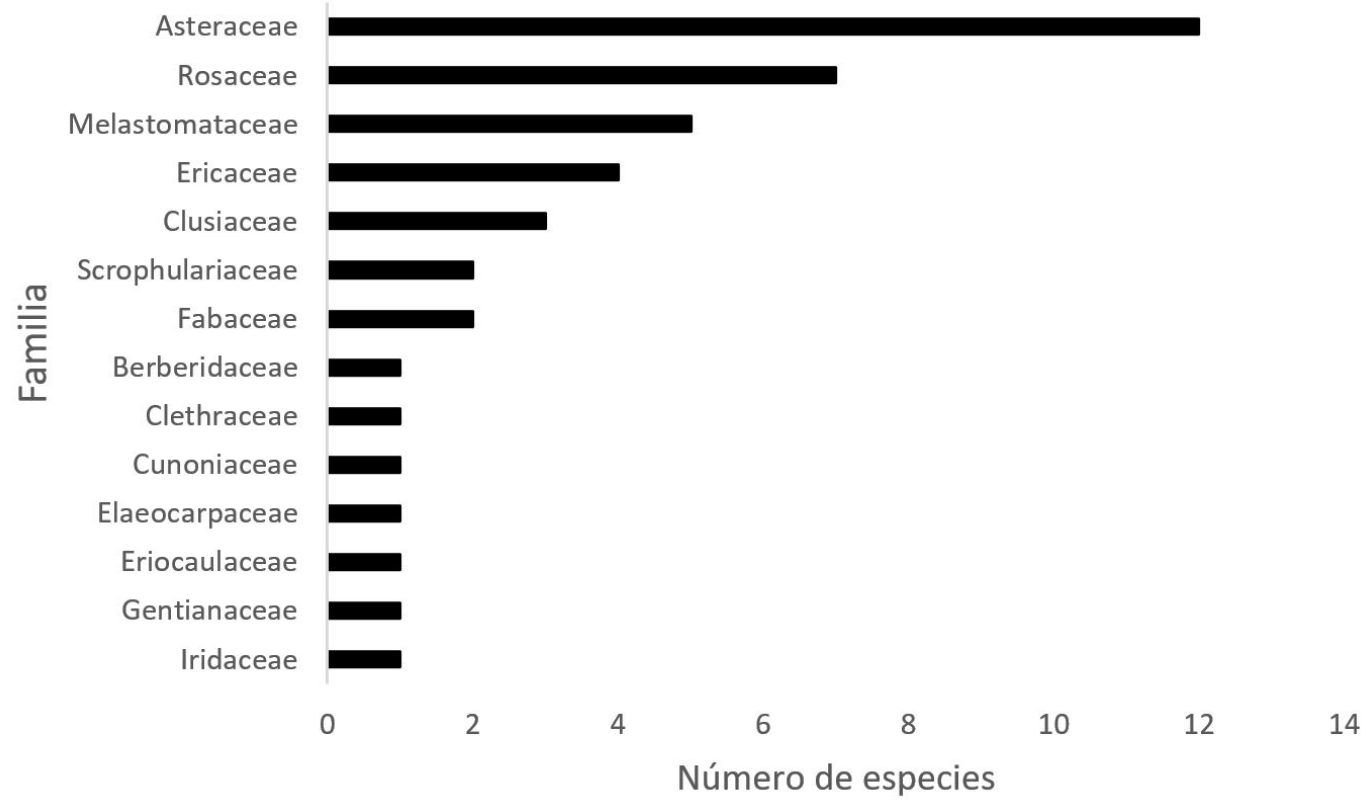

Figura 1. Número de especies de plantas por familia en el predio Aguas Vivas, Soacha-Bogotá, Colombia. 
Tabla 2. Especies de plantas seleccionadas para la evaluación de los rasgos funcionales en el predio Aguas Vivas, Soacha-Bogotá, Colombia

\begin{tabular}{lll}
\hline Familia & Especie & Hábito de crecimiento \\
\hline & Ageratina asclepiadea & Arbusto \\
& Ageratina tinifolia & Arbusto \\
Baccharis prunifolia & Arbusto & Arbusto \\
Asteraceae & Diplostephium rosmarinifolium & Arbusto \\
& Pentacalia ledifosia & Arbusto \\
& Pentacalia pulchella & Arbusto \\
\hline Berberidaceae & Berberis goudotii & Arbusto \\
\hline Clusiaceae & Hypericum juniperinum & Arbusto \\
\hline Elaeocarpaceae & Vallea stipularis & Árbol \\
\hline Fabaceae & Lupinus bogotensis & Hierba \\
\hline Loranthaceae & Gaiadendron punctatum & Árbol \\
\hline & Bucquetia glutinosa & Arbusto \\
Melastomataceae & Miconia elaeoides & Arbusto \\
& Miconia ligustrina & Arbusto \\
\hline Myricaceae & Morella parvifolia & Árbol \\
\hline Myrsinaceae & Myrsine dependens & Árbol \\
\hline Rosaceae & Hesperomeles goudotiana & Árbol \\
\hline Solanaceae & Cestrum buxifolium & Arbusto \\
\hline Symplocaceae & Symplocos theiformis & Árbol \\
\hline
\end{tabular}

Tabla 3. Rasgos foliares evaluados en las 20 especies de plantas seleccionadas en el predio Aguas Vivas, Soacha-Bogotá, Colombia.

\begin{tabular}{llllcl}
\hline Especie & CFMS $(\%)$ & $\mathbf{N}(\%)$ & AF $\left(\mathbf{c m}^{2}\right)$ & AFE $\left(\mathbf{c m}^{2} \cdot \mathbf{g}^{-1}\right)$ & DF $\left(\mathbf{N} . \mathbf{c m}^{-2}\right)$ \\
\hline Ageratina asclepiadea & 66.01 & $2.51 \pm 0.04$ & $41.9 \pm 11.3$ & 61.61 & $0.12 \pm 0.04$ \\
\hline Ageratina tinifolia & 35.04 & $4.86 \pm 0.07$ & $26.88 \pm 4.15$ & 65.56 & $0.04 \pm 0.02$ \\
\hline Baccharis prunifolia & 59.90 & $4.97 \pm 0.09$ & $6.98 \pm 0.71$ & 58.22 & $0.05 \pm 0.018$ \\
\hline Berberis goudotii & 52.23 & $3.63 \pm 0.02$ & $1.75 \pm 0.515$ & 50.17 & $0.34 \pm 0.012$ \\
\hline Bucquetia glutinosa & 48.26 & $3.75 \pm 0.02$ & $3.523 \pm 0.420$ & 74.60 & $0.10 \pm 0.02$ \\
\hline Cestrum buxifolium & 27.27 & $5.48 \pm 0.10$ & $3.828 \pm 0.94$ & 132.00 & $0.10 \pm 0.04$ \\
\hline Diplostephium rosmarinifolium & 85.00 & $2.63 \pm 0.06$ & $0.864 \pm 0.13$ & 50.82 & $0.05 \pm 0.01$ \\
\hline Gaiadendron punctatum & 43.92 & $2.85 \pm 0.02$ & $5.49 \pm 1.058$ & 43.92 & $0.17 \pm 0.03$ \\
\hline Gynoxys fuliginosa & 32.47 & $4.07 \pm 0.2$ & $30.056 \pm 7.25$ & 79.10 & $0.14 \pm 0.04$ \\
\hline Hesperomeles goudotiana & 57.14 & $3.24 \pm 0.02$ & $4.066 \pm 0.51$ & 51.07 & $0.20 \pm 0.07$ \\
\hline Hypericum juniperinum & 90.00 & $3.79 \pm 0.04$ & $0.0076 \pm 0.01$ & 7.71 & $0.3 \pm 0.10$ \\
\hline
\end{tabular}




\begin{tabular}{lllllc}
\hline Lupinus bogotensis & 19.49 & $8.52 \pm 0.01$ & $8.71 \pm 2.5$ & 189.39 & $0.05 \pm 0.01$ \\
\hline Miconia elaeoides & 30.00 & $4.5 \pm 0.06$ & $16.288 \pm 2.42$ & 129.26 & $0.11 \pm 0.03$ \\
\hline Miconia ligustrina & 55.86 & $3.57 \pm 0.04$ & $5.119 \pm 0.94$ & 72.09 & $0.15 \pm 0.03$ \\
\hline Morella parvifolia & 58.33 & $4.17 \pm 0.04$ & $6.019 \pm 0.92$ & 79.19 & $0.11 \pm 0.02$ \\
\hline Myrsine dependens & 69.23 & $3.80 \pm 0.04$ & $0.601 \pm 0.10$ & 66.70 & $0.28 \pm 0.07$ \\
\hline Pentacalia ledifolia & 62.50 & $2.5 \pm 0.21$ & $0.44 \pm 0.08$ & 74.16 & $0.1 \pm 0.01$ \\
\hline Pentacalia pulchella & 52.45 & $3.49 \pm 0.15$ & $2.758 \pm 0.639$ & 86.18 & $0.07 \pm 0.03$ \\
\hline Symplocos theiformis & 33.33 & $3.3 \pm 0.10$ & $4.225 \pm 0.84$ & 65.23 & $0.4 \pm 0.08$ \\
\hline Vallea stipularis & 46.15 & $3.73 \pm 0.01$ & $34.467 \pm 7.23$ & 139.2 & $0.08 \pm 0.03$ \\
\hline
\end{tabular}

Tabla 4. Estadística descriptiva para los rasgos foliares de 20 especies de plantas seleccionadas en el predio Aguas Vivas, SoachaBogotá, Colombia. ST.DEV, desviación estándar; C.V., coeficiente de variación; MIN, valor mínimo; MÁX, valor máximo.

\begin{tabular}{lllllll}
\hline Rasgos foliares & MEDIA & MEDIANA & ST.DEV & C.V. $\%$ & MÍN & MÁX \\
\hline AF $\left(\mathrm{cm}^{2}\right)$ & 10.19 & 4.672 & 12.65 & 124.12 & 0.0076 & 41.9 \\
\hline AFE $\left(\mathrm{cm}^{2} . \mathrm{g}^{-1}\right)$ & 82.58 & 73.125 & 36.94 & 44.73 & 43.92 & 189.39 \\
\hline CFMS $(\%)$ & 51.01 & 52.34 & 18.68 & 36.62 & 19.49 & 90 \\
\hline $\mathrm{N}(\%)$ & 4.03 & 3.75 & 1.33 & 33.12 & 2.4 & 8.52 \\
\hline DF $\left(\mathrm{N} . \mathrm{cm}^{-2}\right)$ & 0.15 & 0.11 & 0.10 & 65 & 0.045 & 0.39 \\
\hline
\end{tabular}

La forma de crecimiento que predominó fue la de tipo arbustivo (70 \% de las especies) y las especies que registraron mayor altura fueron Miconia ligustrina, Vallea stipularis y Gaiadendrom punctatum, con valores de 6, 7 y 8 metros, respectivamente.

Análisis multivariados. En el análisis de componentes principales, los dos primeros componentes explicaron el $72.23 \%$ de la variabilidad total $(49.63 \%$ y $22.6 \%$, respectivamente). En ésta, los valores de AFE y N se asociaron negativamente con el CFMS, de igual manera el AF tuvo una relación negativa con la DF (Figura 2).

A través del análisis clúster, se diferenciaron tres tipos funcionales de acuerdo a sus similitudes descritas en los rasgos de historia de vida (Figura 3).

El TFP1 lo conformaron ocho especies que presentaron en general los valores más bajos de nitrógeno foliar. Sin embargo, este grupo se dividió en dos subclados: el primero con cinco especies (Bucquetia glutinosa, Pentacalia pulchella, Miconia ligustrina, Morella parvifolia y Pentacalia ledifolia) que registraron en promedio valores de AF de $3.57 \pm 2.17 \mathrm{~cm}^{2}$ y CFMS de $55.48 \% \pm 5.45 \%$.
El segundo subclado con tres especies (Diplostephium rosmarinifolium, Myrsine dependens e Hypericum juniperinum) que presentaron las hojas más pequeñas con valores promedio de AF de $0.49 \pm 0.43 \mathrm{~cm}^{2}$ y el más alto contenido de materia seca con $81.41 \pm 10.8 \%$.

El TFP 2 está conformado por ocho especies, todas con estrategia de dispersión por ornitocoria excepto Baccharis prunifolia y con valores intermedios de CFMS y de N. En este tipo funcional se presentan dos subclados, el primero lo conforman las especies Gaiadendrom punctatum, Symplocos theiformis, Hesperomeles goudotiana, Berberis goudotii y Baccharis prunifolia, que registraron el menor valor de AFE con $53.7 \pm 8.1 \mathrm{~cm}^{2} . \mathrm{g}^{-1}$ y otro subclado conformado por Ageratina asclepiadea, Gynoxys fuliginosa y Ageratina tinifolia, especies cuyas hojas fueron las de mayor $\mathrm{AF}$, con $32.9 \pm 7.9 \mathrm{~cm}^{2}$.

En el TFP 3 están las especies Cestrum buxifolium, Miconia elaeoides, Vallea stipularis y Lupinus bogotensis. Todas con hojas de textura membranosa. En este grupo están las especies con mayores valores de contenido de N y AFE y los valores más bajos de CFMS y DF (Tabla 5). 


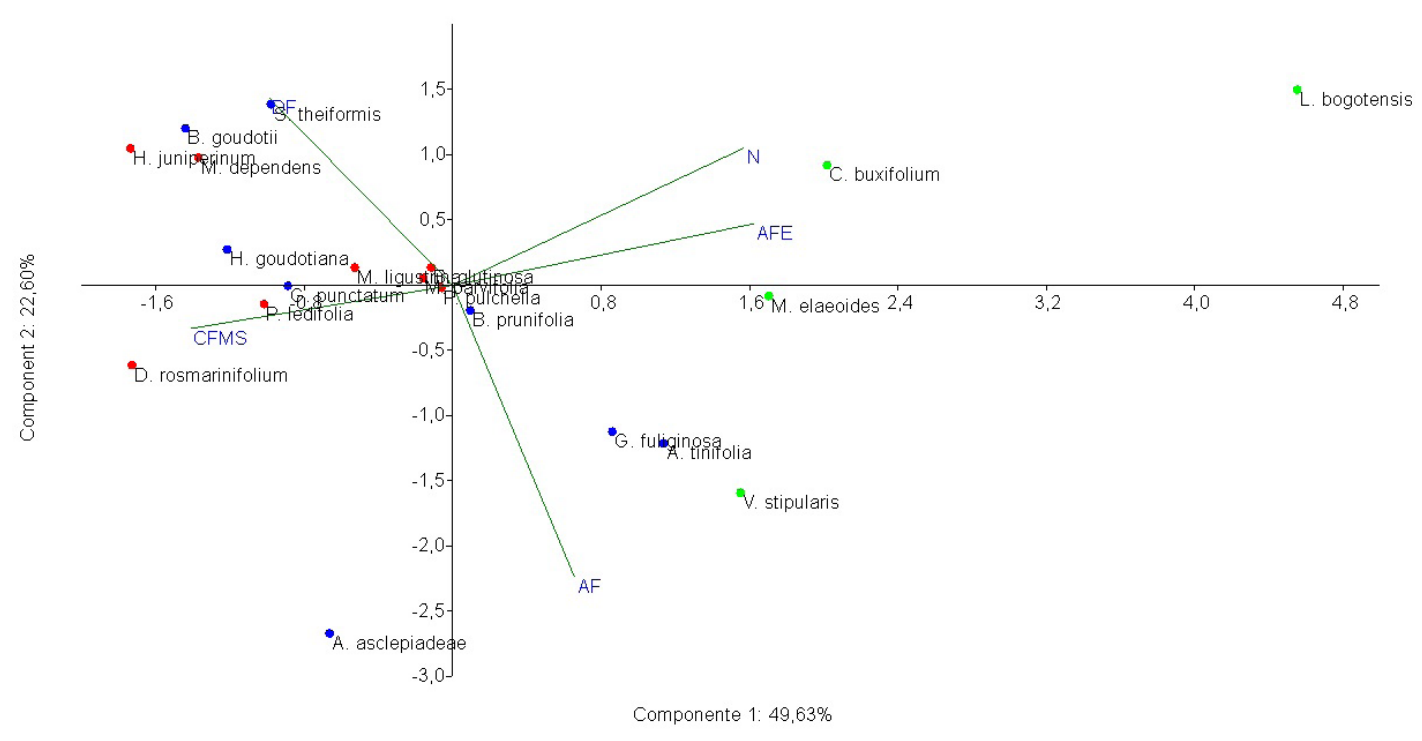

Figura 2. Diagrama de ordenación de las especies de plantas seleccionadas en el predio Aguas Vivas, Soacha-Bogotá, Colombia, en los dos primeros ejes establecidos en el ACP. AF, área foliar; AFE, área foliar específica; CFMS, contenido foliar de materia seca; DF, dureza foliar; N, contenido de nitrógeno. Puntos rojos, TFP1; puntos azules, TFP2; puntos verdes, TFP3. Valor propio eje $1=2.48$; valor propio eje $2=1.12$.

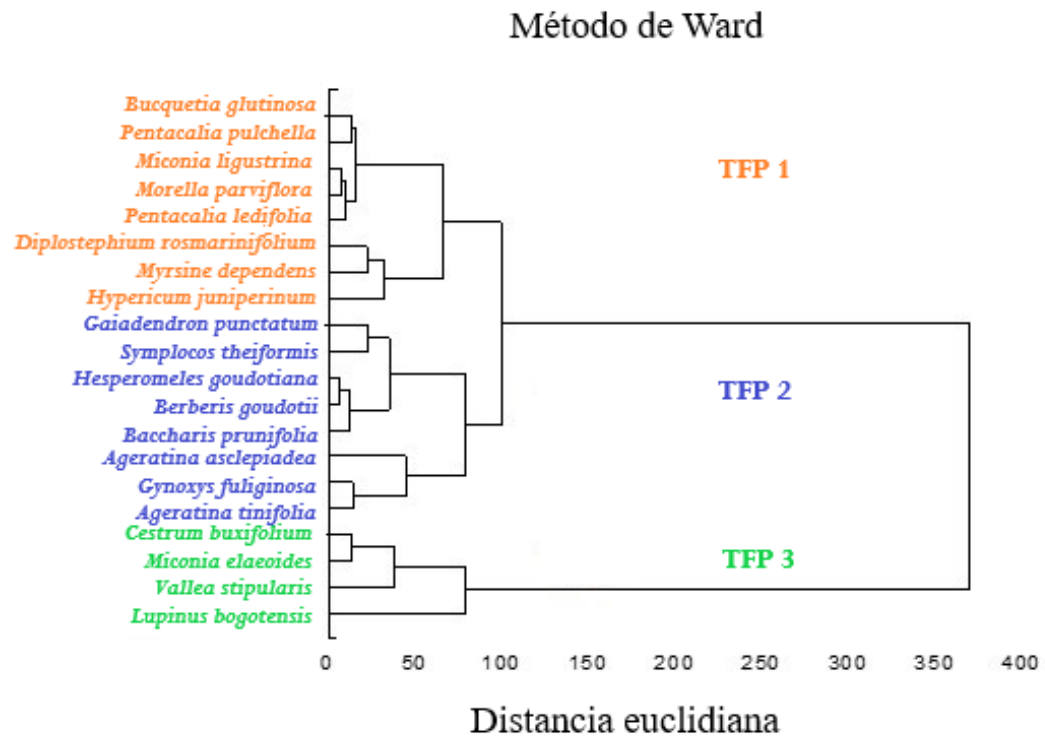

Figura 3. Análisis de conglomerados de plantas seleccionadas en el predio Aguas Vivas, Soacha-Bogotá, Colombia, basado en rasgos categóricos (polinización, dispersión, tipo de fruto, forma de crecimiento, textura de la hoja, tipo de indumento), categóricos cuantitativos (tamaño del fruto, número de semillas por fruto) y cuantitativos (AF, AFE, N, CFMS, DF, AM). 
La prueba T cuadrado de Hotteling mostró diferencias significativas entre los 3 TFP $(a<0.05)$, lo cual indica que existe un agrupamiento de las especies a partir de los valores obtenidos para los diferentes rasgos foliares. En cuanto al análisis discriminante, se encontró que la DF es el rasgo de mayor discriminación entre el TFP1 y TFP2 y entre el TFP1 y TFP3, mientras que para el TFP2 y TFP3 es el N quien diferencia estos grupos (Tabla 6).

Hubo una correlación directa (0.76) entre el AFE y N, que son dos rasgos relacionados con la capacidad de fotosíntesis (Kitajima, 1996). Por otro lado, el N y CFMS se relacionaron negativamente $(-0.56)$. Esto se evidenció en Lupinus bogotensis y Cestrum buxifolium, que fueron las plantas que presentaron mayor contenido de $\mathrm{N}$ y el menor contenido de materia seca en la hoja. El CFMS se relacionó negativamente con el AFE (-0.53), ya que un mayor peso seco da menores valores de AFE (Bermeo, 2010). Esto se representa muy bien en Hypericum juniperinum, que fue la especie con mayor CFMS (90 \%) y menor AFE $\left(0.076 \mathrm{~cm}^{2} \cdot \mathrm{g}^{-1}\right)$. La DF se relacionó negativamente con el AF (-0.36), siendo Myrsine dependens, Berberis goudotii y Symplocos theiformis las especies de mayor dureza foliar, pero que presentaban hojas pequeñas.
El AFE y el AF se correlacionan positivamente; esto se puede deber a que estas dos variables según Kitajima (1996) están correlacionadas con la tasa fotosintética y con el contenido de nitrógeno foliar por unidad de peso foliar. Otra correlación positiva se da entre AF-N y una correlación negativa entre CFMS-AF.

\section{Discusión}

En el TFP1 se encuentran las especies con mayor DF y CFMS y menor N, como Diplostephium rosmarinifolium, Myrsine dependens e Hypericum juniperinum. La DF, puede estar correlacionada con una defensa física más eficaz, puesto que las hojas duras son más difíciles de cortar y digerir por los herbívoros (Barone \& Coley, 2002). Por otro lado, el N puede incidir sobre la intensidad de la herbivoría, sin embargo, esta última también dependerá de la disponibilidad de nutrientes y la edad de las hojas, es decir, cuanto más joven la hoja, mayor será el daño por herbivoría (Coley, 1990).

Tabla 5. Promedio y desviación estándar para los rasgos foliares de cada tipo funcional de plantas (TFP) en el predio Aguas Vivas, Soacha-Bogotá, Colombia. AF, área foliar; AFE, área foliar específica; CFMS, contenido foliar de materia seca; DF, dureza foliar; $\mathrm{N}$, contenido de nitrógeno; $\mathrm{S}$, número de especies.

\begin{tabular}{lllllll}
\hline TFP & $\mathbf{S}$ & AF $\left(\mathrm{cm}^{2}\right)$ & AFE $\left(\mathbf{c m}^{2} \cdot \mathbf{g}^{-1}\right)$ & CFMS $(\%)$ & $\mathbf{N ~ ( \% )}$ & DF $\left(\mathbf{N} . \mathbf{c m}^{-2}\right)$ \\
\hline \multirow{2}{*}{1} & 5 & $3.57 \pm 2.17$ & $77.2 \pm 5.62$ & $55.48 \pm 5.45$ & $3.47 \pm 0.58$ & $0.11 \pm 0.02$ \\
& 3 & $0.49 \pm 0.43$ & $66.9 \pm 16.2$ & $81.41 \pm 10.8$ & $3.40 \pm 0.67$ & $0.24 \pm 0.08$ \\
\hline \multirow{2}{*}{2} & 5 & $4.5 \pm 1.9$ & $53.7 \pm 8.1$ & $48.4 \pm 11.4$ & $3.59 \pm 0.81$ & $0.23 \pm 0.14$ \\
& 3 & $32.9 \pm 7.9$ & $68.7 \pm 9.17$ & $44.5 \pm 18.6$ & $3.77 \pm 1.25$ & $0.10 \pm 0.04$ \\
\hline 3 & 4 & $15.8 \pm 13.4$ & $147.4 \pm 28.2$ & $30.72 \pm 11.20$ & $5.55 \pm 2.1$ & $0.08 \pm 0.02$ \\
\hline
\end{tabular}

Tabla 6. Valores comparativos de la función discriminante para los tres tipos funcionales de plantas (TFP) en el predio Aguas Vivas, Soacha-Bogotá, Colombia, con base en 5 rasgos foliares. AF, área foliar; AFE, área foliar específica; CFMS, contenido foliar de materia seca; $\mathrm{DF}$, dureza foliar; $\mathrm{N}$, contenido de nitrógeno; $\mathrm{S}$, número de especies. Nivel de confianza, $95 \%$.

\begin{tabular}{lllllll}
\hline $\begin{array}{l}\text { Comparación } \\
\text { TFP }\end{array}$ & AF $\left(\mathrm{cm}^{2}\right)$ & AFE $\left(\mathrm{cm}^{2} \cdot \mathbf{g}^{-1}\right)$ & N (\%) & CFMS (\%) & DF $\left(\mathbf{N} . \mathrm{cm}^{-2}\right)$ & P (valor) $\mathbf{\alpha}=0.05$ \\
\hline TFP1 / TFP2 & 0.53591 & -0.48716 & -3.3841 & 0.21382 & $\mathbf{- 2 9 . 5 3 6}$ & 0.0007 \\
\hline TFP1/ TFP3 & -0.39852 & -0.14932 & 3.6536 & 0.15677 & $\mathbf{- 9 . 7 9 3 8}$ & 0.009 \\
\hline TFP2 / TFP3 & -0.76322 & 0.3803 & $\mathbf{7 . 1 1 8}$ & -0.1121 & -0.78502 & 0.0002 \\
\hline
\end{tabular}


El TFP 2 presenta especies con valores bajos de AFE e intermedios de N. Estas especies podrían encajar dentro de la estrategia conservativa, que se caracteriza por ser de sucesión tardía y crecimiento lento (Hobbie, 1992), ya que son especies con AFE baja, la cual se relaciona con la tasa de crecimiento de las plantas (Cornelissen et al, 2003), hojas duras y bajo contenido de nitrógeno (Kühner \& Kleyer, 2008). En un proceso de restauración ecológica, estas especies del TPF 2 podrían plantarse en una etapa posterior al establecimiento de las especies tolerantes a la exposición solar, luego de generarse una cobertura que permita hacer enriquecimientos (Knowles \& Parrotta, 1995).

En el TFP 2, las especies con menor AFE fueron Gaiadendron punctatum $\left(43.92 \mathrm{~cm}^{2} \cdot \mathrm{g}^{-1}\right)$, Berberis goudotii $\left(50.17 \mathrm{~cm}^{2} \cdot \mathrm{g}^{-1}\right)$ y Hesperomeles goudotiana $\left(51.07 \mathrm{~cm}^{2} \cdot \mathrm{g}^{-1}\right)$, lo cual se asocia con hojas más longevas y con más ventajas en hábitats con escasez de nutrientes y agua (Cornelissen et al., 1996).

Tanto en el TFP1 como en el TFP2 se registraron altos valores de DF y CFMS, rasgos presentes en especies que dominan altitudes intermedias (Garnica \& Saldarriaga, 2015), como se reportó en el complejo de Páramo Sumapaz - Cruz Verde y se establecen en áreas de conectividad entre el bosque altoandino y el páramo. Especies con estos rasgos podrían considerarse de crecimiento lento, y de acuerdo a la hipótesis de disponibilidad de recursos de Coley et al. (1985), estas especies invierten más en defensa contra los herbívoros, puesto que están adaptadas a vivir en áreas con pobres condiciones edáficas.

En el TFP 3 están las especies con mayor AFE, como Lupinus bogotensis $\left(189.39 \mathrm{~cm}^{2} . \mathrm{g}^{-1}\right)$, Vallea stipularis $\left(139.2 \mathrm{~cm}^{2} \cdot \mathrm{g}^{-1}\right)$, Cestrum buxifolium $\left(132 \mathrm{~cm}^{2} \cdot \mathrm{g}^{-1}\right) \mathrm{y}$ Miconia elaeoides $\left(129.26 \mathrm{~cm}^{2} \cdot \mathrm{g}^{-1}\right)$. El AFE se relaciona directamente con la capacidad para adquirir energía solar y dióxido de carbono, y por tanto, especies con estas características obtienen una mayor ganancia de carbono, reflejada en altas tasas de crecimiento y mayor capacidad competitiva (Wright et al., 2004). También el AFE podría relacionarse con la longevidad foliar, siendo las las hojas de especies con mayor AFE menos longevas, pues especies con esta característica tienden a invertir una menor cantidad de energía en la defensa de las hojas, pero a cambio de tasas metabólicas más altas (Cornelissen et al., 2003). En este grupo las especies tienen hojas de textura membranosa, son las que presentan mayores valores de contenido $\mathrm{N}$ y los valores más bajos de CFMS, que según Grime et al. (1997) podrían corresponder a especies de estrategia adquisitiva, que se caracterizan por ser colonizadoras, con tasas de crecimiento rápido, de hojas delgadas, suaves, con alta AFE y bajo CFMS. Según Weiher et al. (1999), una tasa de crecimiento rápido se ve representada en áreas foliares grandes, debido a que está relacionada con la estrategia de aprovechamiento de luz (Garnier et al., 2001), y por lo tanto, con una mayor fotosíntesis. Este atributo es importante para la restauración ecológica, ya que las especies con altas tasas de crecimiento tienen la capacidad de acumular biomasa de manera más rápida, y pueden actuar como facilitadoras para el establecimiento de otras especies que requieren determinados microclimas para adaptarse adecuadamente (Gómez \& Vargas, 2011) o como competidoras frente al avance de especies exóticas invasoras en ecosistemas altoandinos, como sucede con Lupinus bogotensis, usada para el control de Ulex europaeus (Vargas et al, 2009). Por otro lado, el alto contenido de nitrógeno en las hojas es una característica que puede emplearse para iniciar procesos de restauración en suelos degradados y susceptibles a la erosión, dado que el $\mathrm{N}$ está relacionado con la tasa de descomposición de la hojarasca la cual aporta rápidamente materia orgánica al suelo (León et al., 2008).

Se ha sugerido que las especies de hojas coriáceas, de alta dureza foliar y bajo contenido de nitrógeno, se desarrollan favorablemente en hábitats donde los suelos son pobres (Loveless, 1962). Tal puede ser el caso de Diplostephium rosmarinifolium, Myrsine dependens, Hypericum juniperinum, Gaiadendrom punctatum, Symplocos theiformis, Hesperomeles goudotiana, Berberis goudotii y Baccharis prunifolia, las cuales presentaron hojas coriáceas, de mayor dureza foliar que las demás especies evaluadas. Este tipo de especies tienden a presentar bajas tasas de crecimiento y por lo tanto a requerir menos nutrientes, lo que se ve reflejado en hojarasca con deficiencia de nutrientes minerales, que se liberan lentamente al bosque (Valladares, 2008).

Las especies de hojas coriáceas presentan las paredes celulares engrosadas y espesas cutículas protectoras, que puede ser una ventaja cuando la planta se encuentra sometida a estrés hídrico, ya que se evita la pérdida de agua (Larcher, 2003); por tal razón, este atributo se podría considerar para la selección de especies que se adapten a condiciones de sequía.

En cuanto a la producción de semillas, algunos autores (Janzen, 1970; Hubbell, 1980; McCanny, 1985) consideran que existe una relación inversa entre semillas producidas y supervivencia de las plántulas. Es decir, 
plantas que producen semillas pequeñas tienen como estrategia de propagación la producción de abundantes semillas para aumentar la probabilidad de supervivencia de algunas plántulas, como puede ocurrir en nuestra área de estudio con las especies de Melastomatacea y Asteraceae. Este carácter es útil para la restauración ecológica, ya que la producción de semillas también puede estar relacionada con la cantidad de dispersores que arriban al lugar, que podría incrementar el grado de conectividad en las zonas a restaurar (Albuquerque et al., 2013).

Por el contrario, en especies como Gaiadendron punctatum, Morella parvifolia, Myrsine dependens y Symplocos theiformis se pueden producir pocas semillas, pero dotadas individualmente de mayor cantidad de reservas (semillas de 5-10 $\mathrm{mm}$ ) que darán lugar a plántulas de mayor tamaño y con mayor probabilidad de sobrevivir (Coomes \& Grubb, 2003). Sin embargo, un tamaño pequeño de semilla también puede permitir que las especies se reproduzcan más rápidamente, inicien procesos sucesionales y colonicen sitios antes que otras especies, mientras que semillas pesadas de tamaño grande tienden a tener un mejor establecimiento cuando compiten con sus vecinos (Weiher et al., 1999).

\section{Conclusión}

Los TFP estuvieron asociados principalmente a variables foliares como el contenido de N, DF y el AFE, más que a la altura de la planta, tipos de fruto, tipo de indumento o hábito de crecimiento. Los rasgos foliares resultaron ser muy informativos a la hora de definir diferentes asociaciones, pues las especies que se identificaron como de rápido crecimiento registraron altos valores de AFE, N, textura de hoja membranosa y bajos contenidos de CFMS tal como se registró en el TFP3 compuesto por Cestrum buxifolium Miconia elaeoides, Vallea stipularis y Lupinus bogotensis. El TFP1 lo conformaron especies con menor AF y mayor CFMS y DF, correlacionándose positivamente entre sí estas dos últimas variables, mientras que el TFP2 se diferenció de los demás tipos por presentar las especies con mayor AF, destacándose el aporte de la familia Asteraceae, pues representaban el 50\% de las especies del grupo. Son necesarios más estudios para evaluar diferentes rasgos funcionales que contribuyan a la recuperación de los ecosistemas en respuesta a las perturbaciones naturales o antrópicas. Características como producción de hojarasca, rasgos radiculares y de la madera, palatabilidad de las hojas, entre otros, se deben considerar a la hora de escoger asociaciones de plantas a incorporar en los diseños; sin embargo, esta selección también se hace teniendo en cuenta las condiciones del sitio a restaurar, el estado de conservación de las especies, las características fisionómico-estructurales de la vegetación presente y los objetivos y metas del proyecto a alcanzar.

\section{Agradecimientos}

Agradecemos al equipo de restauración ecológica y a las profesionales del laboratorio de bromatología de la Subdirección Científica del Jardín Botánico José Celestino Mutis, por el apoyo en el desarrollo de esta investigación.

\section{Referencias}

Albuquerque, L. B., Aquino, F. G., Costa, L. C., Miranda, Z. J. G. \& Sousa, S. R. (2013). Espécies de Melastomataceae Juss. com potencial para restauracão ecológica em área em regeneracão natural para uso potencial na restauracão ecológica de mata ripária no bioma Cerrado. Polibotânica, 35, 1-19.

AOAC -Association of Official Analytical Chemists (2000). Official Methods of Analysis.17 th edition. Washington D. C.: Association of Official Analytical Chemists. 186 pp.

Anderson, E., Marengo, J., Villalba, R., Halloy, S., Young, B., Cordero, D. \& Ruiz, D. (2011). Consequences of climate change for ecosystems and ecosystem services in the tropical Andes. En Herzog, S., Martínez, R., Jorgensen, P., Tiessen, H. (Eds.) Climate Change and Biodiversity in the Tropical Andes: 1-5. Paris, Francia: MacArthur Foundation, Inter-American Institute for Global Change Research (IAI), Scientific Committee on Problems of the Environment (SCOPE).

Batteman, J. (1970). Nutrición Animal. Manual de métodos analíticos. México: Herrero Hermanos, S. A. 468 pp.

Barone, J. \& Coley P. (2002). Herbivorismo y las defensas de las plantas. En: Guariguata M. R. \& G. H. Kattan. (eds.). Ecología y Conservación en bosques Neotropicales. Cartago: Ed. LUR. 465-492 pp.

Bermeo, D. (2010). Determinación y caracterización de tipos funcionales de plantas (TFPs) en bosques secundarios dentro de un gradiente altitudinal y 
su relación con variables bioclimáticas. (Tesis de maestría). Turrialba, Costa Rica: Centro Agronómico Tropical de Investigación y Enseñanza. 126 pp

Castellanos-Castro, C. \& Bonilla, A. (2011). Grupos funcionales de plantas con potencial uso para la restauración en bordes de avance de un bosque altoandino. Acta biológica Colombiana, 16(1), 154-174.

Coley, P. D., Bryant, J. P. \& Chapin F. S.(1985). Resource availability and plant antiherbivore defenses Science, 230, 895-899. https://doi.org/10.1126/science.230.4728.895

Coley, P. D. (1990). Tasas de herbivorismo en diferentes árboles tropicales. En: Leigh, E. G. Jr.; R. A. Stanley, D. M. Windsor, (Eds.). Ecología de un bosque tropical. Balboa, Panamá: The Smithsonian Tropical Research Institute. 191-200 pp.

Coomes, D. \& Grubb, P. (2003). Colonization, tolerance, competition and seed-size variation within functional groups. Trends in Ecology and Evolution, 18, 283-291. https://doi.org/10.1016/S0169-5347(03)00072-7

Cornelissen, J., Castro-Díez, P. \& Hunt, R. (1996). Seedling growth, allocation and leaf attributes in a wide range of woody plant species and types. Journal of Ecology, 84, 755 -765. https:// doi.org/10.2307/2261337

Cornelissen, J., Lavorel, S., Garnier, E., Díaz, S., Buchmann, N., Gurvich, D.E., Reich, P., Steege, H., Morgan, D., Van der Heijden, M.G.A., Pausas, J.G. \& Poorter, H. (2003). Manual mundial para la medición fácil y estandarizada de rasgos funcionales de plantas. Australian Journal of Botany, 51, 335-380. https:// doi.org/10.1071/BT02124

Curtis, J.T. y McIntosh, R.P. (1951). An upland forest continuum in the pariré-forest border region of Wisconsin. Ecology, 32, 476-496. https:// doi.org/10.2307/1931725

Delgado, F. (2012). Clasificación funcional del bosque semideciduo de la Reserva de la Biosfera Península de Guanahacabibes. (Tesis de Doctor en Ciencias). Pinar del Río, Cuba: Universidad de Pinar del Río, Centro de Estudios Forestales. 173 pp.

Díaz, S. \& Cabido, M. (1997). Plant functional types and ecosystem function in relation to global change. Journal of Vegetation Science, 8, 463-474. https:// doi.org/10.2307/3237198

Dunn, E., Shropshire, F., Song, L. \& Mooney, H. (1976). The water factor and convergent evolution in Mediterranean-type vegetation. En: Lange O., Kappen, J. y Schulze, E. (Eds). Water and Plant Life. Pp: 492-505. Berlin: Springer-Verlag. https:// doi.org/10.1007/978-3-642-66429-8_30

Garnica, C. \& Saldarriaga, S. (2015). Diversidad funcional en un gradiente altitudinal del complejo de páramos Sumapaz-Cruz verde. (Tesis de pregrado).
Bogotá D.C.: Universidad Distrital Francisco José de Caldas. 72 pp.

Garnier, E., Laurent, G., Bellman, A., Debain, S., Berthelier, P., Ducout, B., Roumet, C. \& Navas, M.L. (2001). Consistency of species ranking based on functional leaf traits. New Phytologist, 152, 69-83. https:// doi.org/10.1046/j.0028-646x.2001.00239.x

Gitay, H., Noble, I.R. \& Connell, J.H. (1999). Deriving functional types for rain-forest trees. Journal of Vegetation, Science, 10, 641-650. https://doi.org/10.2307/3237079

Gómez, P. \& Vargas, O. (2011). Grupos funcionales de especies promisorias para la restauración ecológica con base en sus rasgos de historia de vida en la Reserva Natural Ibanasca (Ibagué, Tolima, Colombia). En: Vargas O. \& Reyes S. (Eds.). La restauración ecológica en la práctica: Memorias del I Congreso Colombiano de Restauración Ecológica y II Simposio Nacional de Experiencias en Restauración Ecológica. Pp: 239-247. Bogotá D.C.: Universidad Nacional de Colombia.

Granados-Sánchez, D., López-Ríos, F. \& Gama-Flores, J. (1998). Adaptaciones y estrategias de las plantas de zonas áridas. Revista Chapingo Serie Ciencias Forestales y del ambiente, 4(1), 169-178

Grime, J.P., Thompson, K., Hunt, R., Hodgson, J.R., Comelissen, J.H.C., Rorison, I.H., Hendry, G.A.F., Ashenden, T.W., Askew, A.P., Band, S.R., Booth, R.E., Bossard, C.C., Campbell, B.D., Cooper, J.E.L., Davison, A.W., Gupta, P.L., Hall, W., Hand, D.W., Hannah, M.A., Hillier, S.H., Hodkinson, D.J., Jalili, A., Liu, Z., Mackey, J.M.L., Matthews, N., Mowforth, M.A., Neal, A.M., Reader, R.J., Reiling, K., Ross-Fraser, W., Spencer, R.E., Sutton, F., Tasker, D.E., Thorpe, P.C. \& Whitehouse, J.(1997). Integrated screening validates primary axes of specialisation in plants. Oikos, 79, 259-281. https:/ / doi.org/10.2307/3546011

Hammer \& Harper DAT [Internet]. (2005). Paleontological Statistics version 1.34.

Harris, J. \& Harris, W. (1994). Plant Identification Terminology: An Illustrated Glossary. Utah, USA: Spring Lake Publishing. 216 pp.

Hendry, G.A.F. \& Grime, J.P. (1993). Methods in Comparative Plant Ecology: A Laboratory Manual. Londres: Chapman y Hall. 252 pp. https:// doi.org/10.1007/978-94-011-1494-3

Hobbie, S.E. (1992). Effects of plant species on nutrient cycling. Trends in Ecology and Evolution, 7(10), 336339. https://doi.org/10.1016/0169-5347(92)90126-V Holl, K.D. (2002). Effect of shrubs on tree seedling establishment in an abandoned tropical pasture. Journal of Ecology, 90, 179-187. https://doi.org/10.1046/j.0022-0477.2001.00637.x 
Hooper, D. \& Vitousek, P. (1997). The effects of plant composition and diversity on ecosystem processes. Science, 277, 1302-1305. https:// doi.org/10.1126/science.277.5330.1302

Hubbell, S. (1980). Seed predation and the coexistence of tree species in tropical forests. Oikos, 35, 214-229. https:// doi.org/10.2307/3544429

Janzen, D. (1970). Hervibores and the number of tree species in tropical forests. American Naturalist, 104, 501-528. https:// doi.org/10.1086/282687

Kappelle, M. \& Brown, A.D. (2001). Bosques nublados del neotrópico. Santo Domingo de Heredia: Instituto Nacional de Biodiversidad (INBio). 698 pp

Induced Responses to Herbivory. Chicago: University of Chicago Press, 319 pp. https://doi.org/10.7208/chicago/9780226424972.001.0001

Kitajima, K. (1996). Cotyledon functional morphology, patterns of seed reserve utilization and regeneration niches of tropical tree seedlings. En The ecology of tropical forest tree seedlings. Pp 193-210. París: Ed. MD Swaine. https://doi.org/10.1007/978-1-4613-1163-8_19

Kooyman, R. \& Rossetto, M. (2008). Definition of plant functional groups for informing implementation scenarios in resource limited multi-species recovery planning. Biodiversity Conservation, 17, 2917-2937. https://doi.org/10.1007/s10531-008-9405-5

Knowles, O. H. \& Parrotta, J. A. (1995). Amazonian forest restoration: an innovative system for native species selection based on phonological data and field performance indices. Commonwealth Forestry Review, 74, 230-243.

Kühner, A. \& Kleyer, M. (2008). A parsimonious combination of traits predicting plant response to disturbance and soil fertility. Journal of Vegetation Science, 19, 681-692. https:/ / doi.org/10.3170/2008-8-18436

Larcher, W. (2003).Physiological Plant Ecology (fourth edition). New York: Springer-Verlag Berlin Heidelberg. $514 \mathrm{pp}$.

Lavorel, S., Touzard, B., Lebreton, J.D. \& Clément, B. (1998). Identifying functional groups for response to disturbance in an abandoned pasture. Acta Oecológica, 19, 227-240. https://doi.org/10.1016/S1146-609X(98)80027-1

León, J.D., Díez, M.C., Castellanos, J., Osorio, L.F. \& Marín, N. (2008). Grupos funcionales de microorganismos en suelos degradados por minería de aluvión plantados con Acacia mangium. Suelos Ecuatoriales, 38, 75-80.

Loveless, A. R. (1962). Further evidences to support a nutritional interpretation of sclerophylly. Annals of Botany, 26, 549-561.

https://doi.org/10.1093/oxfordjournals.aob.a083814
McCanny, S. (1985). Alternatives in parent-offspring relationships in plants. Oikos, 45, 148-149. https://doi.org/10.2307/3565232

Meli, P., Martínez Ramos, M., Rey Benayas, J.M. \& Carabias, J. (2014). Combining ecological, social and technical criteria to select species for forest restoration. Applied Vegetation Science, 17(4), 744-753. https://doi.org/10.1111/avsc.12096

Mittermeier, R. A. \&. Mittermeier, C. G. (1997). Megadiversity: Earth's Biologically Wealthiest Nations. México: CEMEX. 501 pp.

Montenegro, A. \& Vargas, O. (2008). Caracterización de bordes de bosque altoandino e implicaciones para la restauración ecológica en la Reserva Forestal de Cogua (Colombia). Revista Biología Tropical, 56(3), 1543-1556. https:/ / doi.org/10.15517/rbt.v56i3.5728

Moreno, N. P. (1984). Glosario Botánico Ilustrado. México D.F.: Instituto Nacional de Investigaciones sobre Recursos Bióticos. 300 pp.

Naeem, S., Thompson, L.J., Lawler, S.P., Lawton, J.H. \& Woodfin, R.M. (1994). Declining biodiversity can alter the performance of ecosystems. Nature, 368, 734-737. https:// doi.org/10.1038/368734a0

Nielson, A. \& Griffith, P. (1978). Tissue fixation and staining with osmium tetroxide: the role of phenolic compounds. J. Histochem. Cytochem, 26,138-140. https:/ / doi.org/10.1177/26.2.75221

Niinemets, Ü., Lukjanova. A., Turnbull, M.H. \&Sparrow, A.D. (2007). Plasticity in mesophyll volume fraction modulates light-acclimation in needle photosynthesis in two pines. Tree Physiology, 27, 1137-1151. https://doi.org/10.1093/treephys/27.8.1137

Niklas, K.J., Cobb, E.D., Niinemets, U., Reich, P.B., Sellin, A., Shipley, B. \& Wright, I. J. (2007). “Diminishing returns" in the scaling of functional leaf traits across and within species-groups. Proceedings of the National Academy of Sciences, 104, 8891-8896. https:// doi.org/10.1073/pnas.0701135104

Poorter, L. \& Bongers, F. (2006). Leaf traits are good prediction of plant performance across 53 rain forest species. Ecology, 87(7), 1733-1743. https://doi.org/10.1890/0012-9658(2006)

Rasband, W.S. (2007). ImageJ. US National Institutes of Health, Bethesda, Maryland, USA, http://rsbweb.nih.gov/ij/

Rozendaal, D.M., Hurtado, V.H. \& Poorter. L. (2006). Plasticity in leaf traits of 38 tropical tree species in response to light; relationships with light demand and adult stature. Functional Ecology, 20, 207-216. https:// doi.org/10.1111/j.1365-2435.2006.01105.x Valladares, F. (2008). Ecología del bosque mediterráneo en un mundo cambiante (Segunda edición). Madrid: Ministerio de Medio Ambiente. EGRAF, S. A. 193-230 pp 
Van der Hammen, T. \& González, E. (1960). Holocene and Late Glacial climate and vegetation of Páramo de Palacio (Eastern Coordillera, Colombia, South America). Geologie en Mijnbouw, 39(12), 737 - 746

Van der Hammen, T., Stiles, F.G., Rosselli, L., ChisacaHurtado, A.M., Camargo-Ponce de Leon, G., Guillot, G., Useche, Y. \& Rivera, D. (2008). Protocolo de recuperación y rehabilitación ecológica de humedales en centros urbanos. Bogotá D.C: Secretaria Distrital de Ambiente. 273 pp.

Vargas, O., León, O. \& Díaz, A. (2009). Restauración ecológica en zonas invadidas por retamo espinoso y plantaciones forestales de especies exóticas. Bogotá D.C.: Universidad Nacional de Colombia. 305 pp.
Weiher, E., Van der Werf, A., Thompson, K., Roderick, M., Garnier, E. \& Erksson, O. (1999). Challenging Theophrastus: a common core list of plant traits for functional ecology. Journal of Vegetation Science, 10, 609-620. https:// doi.org/10.2307/3237076

Wright, I.J., Reich, P.B., Westoby, M., Ackerly, D.D., Baruch, Z. \&Bongers, F. (2004). The worldwide leaf economics spectrum. Nature, 428, 821-827. https://doi.org/10.1038/nature02403 
Angélica María Cogollo Calderón

Parques Nacionales Naturales de Colombia

Bogotá, Colombia

angelikmac@gmail.com

https://orcid.org/0000-0001-8707-0930

Patricia Velasco Linares

Bosques \& Semillas SAS

Bogotá, Colombia

patriciavelascolinares@gmail.com

https://orcid.org/0000-0002-5158-0303

\section{Leonardo Manosalva}

Parques Nacionales Naturales de Colombia

Bogotá, Colombia

manosalvaleo@gmail.com

https://orcid.org/000-0002-5008-851X
Caracterización funcional de plantas y su utilidad en la selección de especies para la restauración ecológica de ecosistemas altoandinos

Citación del artículo: Cogollo, A.M., Velasco, P. \& Manoslava, L. (2020). Caracterización funcional de plantas y su utilidad en la selección de especies para la restauración ecológica de ecosistemas altoandinos. Biota Colombiana, 21(1), 1-15. DOI: 10.21068/c2020.v21n01a01.

Recibido: 23 de enero de 2019

Aceptado: 25 de noviembre de 2019 